\title{
Classifying Enteral Nutrition: Tailored for Clinical Practice
}

\author{
Berkeley N. Limketkai ${ }^{1} \cdot$ Neha D. Shah ${ }^{2} \cdot$ Ghias N. Sheikh $^{3} \cdot$ Karen Allen ${ }^{4}$
}

Published online: 31 July 2019

(C) Springer Science+Business Media, LLC, part of Springer Nature 2019

\begin{abstract}
Purpose of Review To discuss the different forms of enteral nutrition, while outlining available evidence for its use in specific conditions and how enteral nutrition composition may or may not influence relevant outcomes.

Recent Findings Enteral nutrition formulas were originally conceived as a liquid form of nutrition for individuals who otherwise could not consume adequate calories through solid food. Over time, the emergence of specialty formulas marketed to benefit specific diseases or conditions has led to a broad range of potentially confusing options. While most options have theoretical benefit for their marketed conditions, the evidence demonstrating practical benefit is not consistent.

Summary Overall, the certainty of evidence for specialty formulas remains low or very low. In most instances, one could begin with standard polymeric formula, except in cases where disease-specific formulas are recommended. Much research is nonetheless still needed to clarify whether some disease-specific formulas are truly beneficial or merely theoretical features.
\end{abstract}

Keywords Enteral nutrition $\cdot$ Elemental formula $\cdot$ Semi-elemental formula $\cdot$ Immunonutrition

\section{Introduction}

Enteral nutrition (EN) involves the administration of a liquid formula into the gastrointestinal tract as an exclusive or partial source of nutrition. EN is often administered via a feeding tube (e.g., nasogastric, nasoduodenal, gastrojejunostomy, jejunostomy), although the term is often used interchangeably with the subclass of oral nutrition supplements (ONS) intended for oral consumption. While EN was originally con-

This article is part of the Topical Collection on Topical Collection on Nutrition and Obesity

Berkeley N. Limketkai

berkeley.limketkai@gmail.com

1 Vatche and Tamar Manoukian Division of Digestive Diseases, UCLA School of Medicine, 100 Medical Center Drive, Suite 345, Los Angeles, CA 90095, USA

2 Comprehensive Cancer Center, Stanford Health Care, Palo Alto, CA, USA

3 Department of Medicine, University of Oklahoma College of Medicine, Oklahoma City, OK, USA

4 Section of Pulmonary, Critical Care \& Sleep Medicine, Veterans Affairs Hospital and University of Oklahoma College of Medicine, Oklahoma City, OK, USA ceived to primarily address the nutritional needs of individuals who could not otherwise consume adequate calories by mouth, specialty EN formulas later emerged on the market to benefit specific diseases or conditions. This has led to a dizzying array of formula options that vary in the type, amount, and complexity of macronutrients (carbohydrates, protein, fats) and micronutrients, caloric density, and osmolarity.

EN formulas can be generally classified as standard (polymeric), peptide-based (elemental or semi-elemental), immune-modulating, disease-specific, and food-based [1]. The classification is primarily based on the protein constituent of the formula with variations based on the overall composition of the formula. Standard polymeric formulas have intact macronutrients and may also have dietary fiber from various sources, such as soy polysaccharides, guar gum, fructooligosaccharides (FOS), and inulin. Normal or near normal digestive and absorptive functions are necessary for the use of polymeric formulas. Elemental formulas contain hydrolyzed ("pre-digested") free amino acids, while semielemental formulas contain oligopeptides. These formulations may also include oligosaccharides and medium-chain triglycerides (MCT) that are theoretically more readily absorbed in the upper gastrointestinal tract, leading to more complete nutrient absorption and less stool residue. Elemental formulas are generally reserved for those who have not tolerated other 
formulas and continue to exhibit symptoms of maldigestion or malabsorption. Immune-modulating and disease-specific formulas are designed for specific diseases or conditions. Depending on the product, the formula may have additions (e.g., omega-3 fatty acids, L-arginine, nucleotides, antioxidants) or restrictions (e.g., carbohydrates for diabetes, lactose, gluten) of specific nutrients to meet needs for disease management and may not necessarily meet the individual's full nutritional needs. An alternative to the synthetic formulas are blenderized foods, which are viewed to provide the full nutritional content and value of real food, but in liquid form; these are often touted by its advocates as more "natural" and "complete."

This review discusses specific conditions that are purported to benefit from EN, while outlining the available evidence for its use in these conditions and how EN composition may or may not influence outcomes.

\section{Gastrointestinal Disorders}

\section{Inflammatory Bowel Disease}

Exclusive enteral nutrition (EEN) involves the use of EN as the predominant source of nutrition, while concurrently abstaining from other food sources. EEN is effective for the induction of remission in Crohn's disease (CD) and is considered a first-line treatment for pediatric CD in Europe [2]. Acceptance of EEN as an intervention has been slow in the United States (US) primarily due to poor tolerability, acceptance, and compliance. An underlying premise of EEN for CD is the reduced exposure of the intestinal mucosa to potentially pro-inflammatory food antigens. A commercial formula with tumor growth factor-beta (TGF- $\beta$ ) has been marketed in Europe and elsewhere for the treatment of CD, but it is not currently available in the US due to legal restrictions on products that claim efficacy for specific diseases without undergoing the traditional drug testing and approval process. Nonetheless, a systematic review by the Cochrane Collaboration found no significant difference in the efficacy of elemental, semi-elemental, or polymeric formulas for the induction of remission in $\mathrm{CD}$, so the need for specialty formulas is unclear [3••]. As for the maintenance of remission in CD, there are still insufficient data on the efficacy and safety of EN.

Given the poor tolerability of EEN, particularly for adults in the long-term, few studies have explored the use of partial enteral nutrition (PEN), where solid foods are permitted in addition to EN for consumption. An observational open-label, uncontrolled cohort study of 47 children and young adults with CD showed that PEN-where up to $50 \%$ of calories derived from polymeric EN and the remainder of calories from an exclusion diet - induced remission in $70 \%$ of participants [4]. An RCT of 50 children with CD later found EN to be superior to PEN for the induction of remission (42\% vs. $15 \% ; P=0.04)$ [5]. Briefly, there is currently insufficient evidence at present to recommend PEN for the induction of remission in $\mathrm{CD}$.

For ulcerative colitis (UC), an early case series has suggested EEN to be helpful, although prospective studies are grossly lacking [6].

\section{Gastrointestinal Intolerance and Malabsorption}

Fiber-containing formulas have been used in the management of diarrhea presumably resulting from EN administration. However, the evidence to support this practice is currently unclear. An earlier meta-analysis of 7 randomized controlled trials (RCT) with 400 patients on EN did not find dietary fiber to improve diarrhea, although a subgroup analysis revealed benefit in non-critically ill patients [7]. A more recent metaanalysis from 2015 of 26 RCT and observational studies found fiber to reduce diarrhea in patients on EN [8]. Similar to the earlier meta-analysis, subgroup analyses revealed that this benefit was only seen in non-critically ill patients. The lack of benefit from fiber in the critically ill population may stem from multi-factorial insults, such as antibiotic use, intestinal dysbiosis, intestinal inflammation, and loss of intestinal mucosal integrity.

Fibers in EN formulas usually comprise soy polysaccharides and guar gum, although newer formulas may have fiber in the form of FOS and inulin, which are fermentable oligosaccharides. Interestingly, fermentable oligo-, di-, monosaccharides, and polyols (FODMAPs) have recently garnered attention as a potential contributor to diarrhea while on EN and in general [9]. Dietary reduction of FODMAPs has been demonstrated across RCTs to improve symptoms of irritable bowel syndrome (IBS) [10•]. As such, EN formulas low in FODMAPs have been marketed for individuals with digestive intolerances, discomfort, or IBS-associated symptoms. An RCT of 84 patients who were administered EN with low-, moderate-, and high-FODMAP content for 14 days revealed greater improvement in diarrheal symptoms (among those with baseline diarrhea) in the low-FODMAP group [11].

Elemental and semi-elemental formulas are also often used in clinical practice for individuals with symptoms suspected from intestinal malabsorption. The premise of this practice stems from the concept that pre-digested macronutrients in their simplest forms are more readily absorbed and lead to less stool output. While this is a logical assumption to support the use of peptide-based formulas in patients with diarrhea, there are no clear data to suggest that this strategy is generally effective.

Patients with short bowel syndrome could potentially be candidates for these formulas due to significant risk of 
malabsorption. However, a small study of 7 patients with end jejunostomies found no benefit of a peptide-based formula over a polymeric one [12]. Patients would nonetheless benefit from polymeric formulas, as they have been shown to augment intestinal adaptation $[13,14]$. The 2016 guidelines from the European Society for Clinical Nutrition and Metabolism (ESPEN) recommend the use of isotonic polymeric EN formulas to promote intestinal adaptation in short bowel syndrome [15].

Specialty formulas have also been marketed with hypoallergenic variants for individuals with specific or unclear food allergies. Their compositions may include an elemental formulation and may also exclude lactose, soy, and intact cow's milk protein, food components that have typically been associated with food allergies. Although not specifically marketed as being hypoallergenic, there are other enteral formulas from reputable manufacturers that specifically exclude certain substances, such as gluten, whey, casein, egg, and soy. There are currently no rigorous studies evaluating the efficacy of these formulas for patients with gastrointestinal sensitivity or intolerance.

\section{Pancreatitis}

The use of immunomodulating formulas have been explored for acute pancreatitis. These formulas are primarily comprised of the standard EN mixture and supplemented with L-glutamine, L-arginine, nucleotides, and omega-3 polyunsaturated fatty acids (PUFAs). The premise of these immunomodulating formulas involves the attenuation of the inflammatory response that subsequently reduces the self-perpetuating cycle of pancreatic autolysis and inflammation-provoking injury. In a small study of 32 patients with acute pancreatitis, a Chinese group revealed that early EN containing glutamine and arginine had lower intestinal permeability and serum endotoxin levels [16]. A small RCT of 28 patients with moderate-severe acute pancreatitis showed that exogenous omega-3 PUFA reduced the time of jejunal feeding and LOS [17]. However, a meta-analysis of 3 RCTs comparing immunonutrition with standard EN did not show a difference in the risk of total infectious complications, death, or length of stay in patients with acute pancreatitis [18]. A subsequently larger meta-analysis of 12 RCTs showed that glutamine supplementation reduced infectious complications and mortality, but not length of stay, in patients with acute pancreatitis [19]. However, subgroup analyses revealed that this benefit was only present among patients who received parenteral nutrition in addition to EN. The authors therefore concluded that glutamine supplementation with EN is not required for acute pancreatitis.

\section{Chyle Leaks}

In the nutritional management of chyle leaks, there is nearcomplete restriction of long-chain triglycerides either through a fat-free diet or the administration of elemental EN formulas. MCT-containing elemental formulas are often utilized for the short term ( 2 weeks) to decrease chyle output and promote closure. If the leak persists, parenteral nutrition is indicated. In earlier case reports, successful treatment of chylous fistulas was done with administration of MCT-containing enteral formulas [20]. In a retrospective review of 245 patients that underwent pancreatic resection, 40 developed a chyle leak while on EN. The patients were later switched to an MCTcontaining EN formula that ultimately led to the resolution of the chyle leaks [21]. None of these patients required further surgical intervention or parenteral nutrition.

\section{Hepatic Disorders}

Polymeric formulas often suffice for patients with hepatic disease, particularly those who cannot meet their nutritional needs by mouth. In the presence of ascites, formulas with a higher caloric density and fluid restriction are preferred to reduce the volume of intake. Consumption of ONS is initially recommended, but fine-bore nasogastric or nasoduodenal delivery would be appropriate - even in the presence of esophageal varicesfor patients unable to consume adequate oral intake [22]. Gastrostomy tubes are not recommended in patients with ascites.

For patients who develop hepatic encephalopathy, enteral formulas enriched in branched-chain amino acids (valine, leucine, isoleucine) have been proposed, as these amino acids less readily cross the blood-brain barrier. In an early randomized trial of 37 patients with protein-intolerant hepatic cirrhosis, those who received a branched-chain amino acid solution had lower risk of developing encephalopathy than those who received dietary protein [23]. A subsequent systematic review by the Cochrane Collaboration that included 16 RCTs among 827 participants found branched-chain amino acids improved hepatic encephalopathy; the data for this outcome were graded as high-certainty evidence [24]. This observation persisted even when excluding trials with lactulose or neomycin, and a separate analysis found no difference between branchedchain amino acids and lactulose or neomycin. There was however no effect on nutritional parameters, quality of life, or mortality, and the investigators concede that further investigation is still needed to more rigorously evaluate these outcomes. Joint guidelines from American Society for Parenteral and Enteral Nutrition (ASPEN) and the Society of Critical Care Medicine (SCCM) do not recommend routine use of branched-chain amino acid formulas above standard 
formulations, citing no evidence of added benefit for critically ill patients with encephalopathy [25•].

\section{Diabetes}

Nutrition and pharmacologic approaches to glycemic control during EN administration are based on several factors, such as the degree of hyperglycemia, presence of critical illness, carbohydrate content in the regimen, and pattern of delivery (continuous, bolus, or nocturnal). To improve hyperglycemia, diabetes-specific formulas have a lower carbohydrate provision with slowly digestible forms of carbohydrates (e.g., isomaltose, oligosaccharides, fructose, and source of starch) and a higher concentration of fats as monounsaturated fats. Dietary fiber may be present in the form of FOS and soy fiber.

The effectiveness of diabetes-specific formulas in the glycemic management in diabetic patients is currently unclear. An early meta-analysis of 6 RCTs with 203 participants with type II diabetes similarly found that diabetes-specific formulas, when compared with standard formulas, led to a lower post-prandial rise in serum glucose concentrations; 2 RCTs with 44 participants showed diabetes-specific formulas to lead to lower peak blood glucose concentrations [26]. Several small studies subsequently confirmed diabetes-specific formulas reduced post-prandial glucose and mean glucose levels in non-hospitalized patients with type II diabetes [27-29]. An RCT in 55 patients with insulin-treated type II diabetes and neurologic disorders requiring long-term EN found that those receiving diabetes-specific formulas also had lower insulin requirements, fasting and afternoon glucose levels, and hemoglobin A1C than those receiving standard formulas [30]. On the other hand, the 2013 guidelines from the ASPEN graded the certainty of evidence as low and could not make a formal recommendation for diabetes-specific formulas [31]. In the clinical setting, we would recommend beginning with standard formulas and consider diabetes-specific formulas if glycemic control significantly worsens or remains poor.

\section{Obesity}

EN formulas designed for obese patients are often referred to as bariatric formulas and are considered to be standard formulas. These formulas are generally hypocaloric, while including a higher provision of protein. The rationale when using these formulas is the avoidance of excess calories, while providing adequate proteins for healing, recovery, and preservation of muscle mass. The formulas can also be used to promote intentional weight loss. The majority of studies on the use of hypocaloric, high-protein enteral formulas have focused on the critically ill obese patient population. The ASPEN clinical guidelines weakly recommend a trial of hypocaloric, highprotein feeding in the hospitalized obese patient without significant renal or hepatic dysfunction [32]. Hypocaloric feeds can begin at 50 to $70 \%$ of estimated caloric requirements with a protein provision of $1.2 \mathrm{~g} / \mathrm{kg}$ of actual body weight or 2 to $2.5 \mathrm{~g} / \mathrm{kg}$ of ideal body weight. Importantly, hypocaloric, lowprotein feeds should be avoided, as they are associated with poor outcomes. The overall certainty of evidence was considered low, and further research is needed on nutrition support in this patient population.

\section{Renal Disorders}

EN formulas for chronic kidney disease (CKD) are diseasespecific formulas that include a calorie-dense formula with minimization of fluid volume and a lower provision of specific electrolytes (sodium, potassium, phosphorus). For patients with $\mathrm{CKD}$ on dialysis, formulas include a higher provision of protein to account for protein losses from dialysis. There are currently no specialized EN formulas for acute renal insufficiency.

Data on the efficacy of disease-specific formulas are currently sparse. An early systematic review found 2 RCTs that compared disease-specific with standard formulas in 119 participants on hemodialysis [33]. The investigators concluded there was insufficient evidence to compare these formulas. ESPEN guidelines from 2006 provide grade $\mathrm{C}$ recommendations to use standard enteral formulas for the majority of patients with acute renal failure [34]. ESPEN nonetheless notes that disease-specific formulas can be considered in patients with acute renal failure who experience electrolyte derangements. For patients with CKD, standard formulas can be used up to 5 days. When used greater than 5 days, disease-specific formulas could be considered. For patients undergoing hemodialysis, hemodialysis-specific formulas with higher protein and lower potassium and phosphorus content should be used. ASPEN guidelines from 2010 provide grade D recommendations that protein intake be adjusted based on the extent of renal function and losses via dialysis. The ASPEN guidelines are however silent on the choice of disease-specific or standard formulas. More research is still needed to clarify the benefit of disease-specific formulas in renal disorders.

\section{Pulmonary Disorders and Critical Care}

A disease-specific high-fat and low-carbohydrate formula had long been preferred for patients suffering from hypercapnic respiratory failure, such as from chronic obstructive pulmonary disease exacerbations, obesity hypoventilation syndrome, and neuromuscular disorders. The theoretical benefit stems from the fact that the respiratory quotient $\left(\mathrm{CO}_{2}\right.$ produced $/ \mathrm{O}_{2}$ consumed $)$ is higher for carbohydrates (1) than fats $(0.7)$; therefore, patients 
with impaired ability to excrete $\mathrm{CO}_{2}$ secondary to respiratory failure would fare better with a higher fat enteral regimen. While a small study demonstrated that higher fat led to decreased mechanical ventilation days [35], these results have not yet been replicated [36]. Therefore, due to lack of clinical benefit in failing to reduce $\mathrm{PaCO}_{2}$ during ventilator weaning [37], high-fat formulas are no longer recommended. In fact, calorie-dense fluid-restricted formulas are currently considered most efficacious in pulmonary failure [38].

The recent literature has focused on whether trophic or full EN is superior. Trophic feeding has been loosely defined as 400 to $800 \mathrm{kcal} /$ day, providing 25 to $40 \%$ of the daily caloric goal. The EDEN Trial looked at patients with acute respiratory distress syndrome (ARDS) in the first 6 days of mechanical ventilation and compared various outcomes including infection, organ failure-free days, ventilator-free days, and 60-day mortality between two groups who were given either trophic ( $400 \mathrm{kcal} /$ day $)$ or full (1300 kcal/day) feeding [39]. The study did not detect any difference between the groups. A 1-year follow-up study of the EDEN Trial also failed to show any difference between the two groups with regard to arm anthropometrics, strength, pulmonary function, 6-min-walk distance, and cognitive function [40]. The PERMIT Trial incorporated a broader base of critically ill patients not limited to ARDS and looked at groups receiving either trophic feeding for up to 14 initial days or full standard feeding [41]. There was no difference in 90-day mortality between the groups. Neither trial featured undernourished patients, leading to a post hoc analysis of the PERMIT trial [42], which again failed to show worse outcomes in the trophic feeding group with a high NUTRIC score (a scale to quantify risk of experiencing malnutrition). An observational study has since linked improved nutrition in high NUTRIC score patients to lower mortality and faster discharge [43॰], but additional studies are required.

The anti-inflammatory properties of omega-3 fatty acids in EN were previously shown to benefit ARDS, specifically improving oxygenation and decreasing intensive care unit (ICU) length of stay and duration of mechanical ventilation [44]. However, these trials suffered by also giving the control groups omega- 6 fatty acids, which have pro-inflammatory properties, thus skewing outcome data. This led to the development of the OMEGA trial, the largest trial to evaluate omega-3 fatty acid efficacy in ARDS [45]. The study showed that patients who received omega- 3 fatty acids had fewer ICU-free and ventilator-free days, although the study was ultimately stopped early due to futility, as it was not projected to reach statistical significance in the primary outcome. A later meta-analysis of seven trials also failed to show mortality benefit or fewer ventilator or ICU-free days among patients receiving omega-3 fatty acid supplementation [46]. The latest joint guidelines from ASPEN and the SCCM recommend a standard polymeric isotonic formula [25]. In critically ill patients, early initiation of higher protein nutrition, matching at least $80 \%$ of the prescribed intake, has been shown to lead to improved survival and shorter ICU stays, a link that has not been proven with greater energy intake (when controlled for protein) [47].

Lastly, while EN is often started with patients on vasopressors, the recommendation is to do so cautiously and only on low-to-moderate doses. The basis of this is the NUTRIREA-2 trial [48•], which showed increased bowel ischemia incidence as a secondary outcome in shock patients on high-dose vasopressors who were given EN.

\section{Conclusion}

There is a broad array of choices for EN formulas. In most cases, the use of a standard polymeric formula would suffice. However, in specific disease states, peptide-based or disease-specific formulas may play a role. For patients with gastrointestinal intolerance or malabsorptive issues, a fiber-containing, peptide-based, or low-FODMAP formula could be considered. While EEN is helpful for $\mathrm{CD}$, there is so far no evidence that specialized formulas provide any benefit beyond the standard polymeric formula. In liver disease, EN plays a significant role in helping maintain adequate nutritional intake. While the formulas enriched in branchedchain amino acid may help reduce hepatic encephalopathy in some studies, society guidelines note the lack of evidence in critically ill patients. For hyperglycemia and diabetes, diabetes-specific formulas can be considered if glycemic control remains poor. With CKD, European guidelines recommend the use of disease-specific formulas in the appropriate context, while American guidelines are so far silent. For pulmonary disorders, calorie-dense fluid-restricted formulas are preferred. In critically ill patients, standard polymeric formulas can be used. More importantly, EN even at trophic feeding rates should be considered. Overall, the certainty of evidence for disease-specific formulas remains very low or low; in most instances, one could begin with the standard polymeric formula, except in cases where diseasespecific formulas are recommended. Much research is nonetheless still needed to clarify whether some disease-specific formulas are truly beneficial or merely theoretical features. 


\section{References}

Papers of particular interest, published recently, have been highlighted as:

- Of importance

- Of major importance

1. Lochs H, Allison SP, Meier R, Pirlich M, Kondrup J, Schneider S, et al. Introductory to the ESPEN guidelines on enteral nutrition: terminology, definitions and general topics. Clin Nutr. 2006;25(2): 180-6. https://doi.org/10.1016/j.clnu.2006.02.007.

2. Forbes A, Escher J, Hebuterne X, Klek S, Krznaric Z, Schneider S, et al. ESPEN guideline: clinical nutrition in inflammatory bowel disease. Clin Nutr. 2017;36(2):321-47. https://doi.org/10.1016/j. clnu.2016.12.027.

3.• Narula N, Dhillon A, Zhang D, Sherlock ME, Tondeur M, Zachos M. Enteral nutritional therapy for induction of remission in Crohn's disease. Cochrane Database Syst Rev. 2018;4:CD000542. https:// doi.org/10.1002/14651858.CD000542.pub3 Systematic review and meta-analysis demonstring the efficacy of exclusive enteral nutrition for Crohn's disease.

4. Sigall-Boneh R, Pfeffer-Gik T, Segal I, Zangen T, Boaz M, Levine A. Partial enteral nutrition with a Crohn's disease exclusion diet is effective for induction of remission in children and young adults with Crohn's disease. Inflamm Bowel Dis. 2014;20(8):1353-60. https://doi.org/10.1097/MIB.0000000000000110.

5. Johnson T, Macdonald S, Hill SM, Thomas A, Murphy MS. Treatment of active Crohn's disease in children using partial enteral nutrition with liquid formula: a randomised controlled trial. Gut. 2006;55(3):356-61. https://doi.org/10.1136/gut.2004.062554.

6. Ricour C, Duhamel JF, Nihoul-Fekete C. Use of parenteral and elementary enteral nutrition in the treatment of Crohn's disease and ulcerative colitis in children. Arch Fr Pediatr. 1977;34(6): 505-13.

7. Yang G, Wu XT, Zhou Y, Wang YL. Application of dietary fiber in clinical enteral nutrition: a meta-analysis of randomized controlled trials. World J Gastroenterol. 2005;11(25):3935-8.

8. Kamarul Zaman M, Chin KF, Rai V, Majid HA. Fiber and prebiotic supplementation in enteral nutrition: a systematic review and metaanalysis. World J Gastroenterol. 2015;21(17):5372-81. https://doi. org/10.3748/wjg.v21.i17.5372.

9. Halmos EP. Role of FODMAP content in enteral nutritionassociated diarrhea. J Gastroenterol Hepatol. 2013;28(Suppl 4): 25-8. https://doi.org/10.1111/jgh. 12272.

10. Schumann D, Klose P, Lauche R, Dobos G, Langhorst J, Cramer H. Low fermentable, oligo-, di-, mono-saccharides and polyol diet in the treatment of irritable bowel syndrome: a systematic review and meta-analysis. Nutrition. 2018;45:24-31. https://doi.org/10.1016/j. nut.2017.07.004 Systematic review and meta-analysis of the benefit of the low FODMAP diet for symptoms of irritable bowel syndrome.

11. Yoon SR, Lee JH, Lee JH, Na GY, Lee KH, Lee YB, et al. LowFODMAP formula improves diarrhea and nutritional status in hospitalized patients receiving enteral nutrition: a randomized, multicenter, double-blind clinical trial. Nutr J. 2015;14:116. https://doi. org/10.1186/s12937-015-0106-0.

12. McIntyre PB, Fitchew M, Lennard-Jones JE. Patients with a high jejunostomy do not need a special diet. Gastroenterology. 1986;91(1):25-33.

13. Levy E, Frileux P, Sandrucci S, Ollivier JM, Masini JP, Cosnes J, et al. Continuous enteral nutrition during the early adaptive stage of the short bowel syndrome. Br J Surg. 1988;75(6):549-53.

14. Joly F, Dray X, Corcos O, Barbot L, Kapel N, Messing B. Tube feeding improves intestinal absorption in short bowel syndrome patients. Gastroenterology. 2009;136(3):824-31. https://doi.org/ 10.1053/j.gastro.2008.10.084.

15. Pironi L, Arends J, Bozzetti F, Cuerda C, Gillanders L, Jeppesen $\mathrm{PB}$, et al. ESPEN guidelines on chronic intestinal failure in adults. Clin Nutr. 2016;35(2):247-307. https://doi.org/10.1016/j.clnu. 2016.01.020.

16. Huang XX, Wang XP, Ma JJ, Jing DD, Wang PW, Wu K. Effects of enteral nutrition supplemented with glutamine and arginine on gut barrier in patients with severe acute pancreatitis: a prospective randomized controlled trial. Zhonghua Yi Xue Za Zhi. 2008;88(34): 2407-9.

17. Lasztity N, Hamvas J, Biro L, Nemeth E, Marosvolgyi T, Decsi T, et al. Effect of enterally administered n-3 polyunsaturated fatty acids in acute pancreatitis-a prospective randomized clinical trial. Clin Nutr. 2005;24(2):198-205. https://doi.org/10.1016/j.clnu. 2004.12.008.

18. Petrov MS, Atduev VA, Zagainov VE. Advanced enteral therapy in acute pancreatitis: is there a room for immunonutrition? A metaanalysis. Int J Surg. 2008;6(2):119-24. https://doi.org/10.1016/j. ijsu.2008.01.003.

19. Asrani V, Chang WK, Dong Z, Hardy G, Windsor JA, Petrov MS. Glutamine supplementation in acute pancreatitis: a meta-analysis of randomized controlled trials. Pancreatology. 2013;13(5):468-74. https://doi.org/10.1016/j.pan.2013.07.282.

20. Martin IC, Marinho LH, Brown AE, McRobbie D. Medium chain triglycerides in the management of chylous fistulae following neck dissection. Br J Oral Maxillofac Surg. 1993;31(4):236-8.

21. Abu Hilal M, Layfield DM, Di Fabio F, Arregui-Fresneda I, Panagiotopoulou IG, Armstrong TH, et al. Postoperative chyle leak after major pancreatic resections in patients who receive enteral feed: risk factors and management options. World J Surg. 2013;37(12): 2918-26. https://doi.org/10.1007/s00268-013-2171-x.

22. Plauth M, Cabre E, Riggio O, Assis-Camilo M, Pirlich M, Kondrup J, et al. ESPEN guidelines on enteral nutrition: liver disease. Clin Nutr. 2006;25(2):285-94. https://doi.org/10.1016/j.clnu.2006.01.018.

23. Horst D, Grace ND, Conn HO, Schiff E, Schenker S, Viteri A, et al. Comparison of dietary protein with an oral, branched chainenriched amino acid supplement in chronic portal-systemic encephalopathy: a randomized controlled trial. Hepatology. 1984;4(2): 279-87.

24. Gluud LL, Dam G, Les I, Marchesini G, Borre M, Aagaard NK, et al. Branched-chain amino acids for people with hepatic encephalopathy. Cochrane Database Syst Rev. 2017;5:CD001939. https:// doi.org/10.1002/14651858.CD001939.pub4.

25. McClave SA, Taylor BE, Martindale RG, Warren MM, Johnson $\mathrm{DR}$, Braunschweig C, et al. Guidelines for the provision and assessment of nutrition support therapy in the adult critically ill patient: Society of Critical Care Medicine (SCCM) and American Society for Parenteral and Enteral Nutrition (A.S.P.E.N.). JPEN J Parenter Enteral Nutr. 2016;40(2):159-211. https://doi.org/10. $1177 / 0148607115621863$ Guidelines for the use of enteral nutrition in critical illness.

26. Elia M, Ceriello A, Laube H, Sinclair AJ, Engfer M, Stratton RJ. Enteral nutritional support and use of diabetes-specific formulas for patients with diabetes: a systematic review and meta-analysis. Diabetes Care. 2005;28(9):2267-79.

27. Lansink M, Hofman Z, Genovese S, Rouws C, Ceriello A. Improved glucose profile in patients with type 2 diabetes with a new, high-protein, diabetes-specific tube feed during 4 hours of continuous feeding. JPEN J Parenter Enteral Nutr. 2017;41(6): 968-75. https://doi.org/10.1177/0148607115625635.

28. Alish CJ, Garvey WT, Maki KC, Sacks GS, Hustead DS, Hegazi RA, et al. A diabetes-specific enteral formula improves glycemic variability in patients with type 2 diabetes. Diabetes Technol Ther. 2010;12(6):419-25. https://doi.org/10.1089/dia.2009.0185. 
29. Ceriello A, Lansink M, Rouws CH, van Laere KM, Frost GS. Administration of a new diabetes-specific enteral formula results in an improved $24 \mathrm{~h}$ glucose profile in type 2 diabetic patients. Diabetes Res Clin Pract. 2009;84(3):259-66. https://doi.org/10. 1016/j.diabres.2009.02.013.

30. Pohl M, Mayr P, Mertl-Roetzer M, Lauster F, Haslbeck M, Hipper $\mathrm{B}$, et al. Glycemic control in patients with type 2 diabetes mellitus with a disease-specific enteral formula: stage II of a randomized, controlled multicenter trial. JPEN J Parenter Enteral Nutr. 2009;33(1):37-49. https://doi.org/10.1177/0148607108324582.

31. McMahon MM, Nystrom E, Braunschweig C, Miles J, Compher C, American Society for P, et al. A.S.P.E.N. clinical guidelines: nutrition support of adult patients with hyperglycemia. JPEN J Parenter Enteral Nutr. 2013;37(1):23-36. https://doi.org/10.1177/ 0148607112452001

32. Choban P, Dickerson R, Malone A, Worthington P, Compher C, American Society for P, et al. A.S.P.E.N. clinical guidelines: nutrition support of hospitalized adult patients with obesity. JPEN J Parenter Enteral Nutr. 2013;37(6):714-44. https://doi.org/10.1177/ 0148607113499374.

33. Stratton RJ, Bircher G, Fouque D, Stenvinkel P, de Mutsert R, Engfer M, et al. Multinutrient oral supplements and tube feeding in maintenance dialysis: a systematic review and meta-analysis. Am J Kidney Dis. 2005;46(3):387-405. https://doi.org/10.1053/j.ajkd. 2005.04.036.

34. Cano N, Fiaccadori E, Tesinsky P, Toigo G, Druml W, Dgem, et al. ESPEN guidelines on enteral nutrition: adult renal failure. Clin Nutr. 2006;25(2):295-310. https://doi.org/10.1016/j.clnu.2006.01.023.

35. al-Saady NM, Blackmore CM, Bennett ED. High fat, low carbohydrate, enteral feeding lowers $\mathrm{PaCO} 2$ and reduces the period of ventilation in artificially ventilated patients. Intensive Care Med. 1989;15(5):290-5.

36. Mesejo A, Acosta JA, Ortega C, Vila J, Fernandez M, Ferreres J, et al. Comparison of a high-protein disease-specific enteral formula with a high-protein enteral formula in hyperglycemic critically ill patients. Clin Nutr. 2003;22(3):295-305.

37. van den Berg B, Bogaard JM, Hop WC. High fat, low carbohydrate, enteral feeding in patients weaning from the ventilator. Intensive Care Med. 1994;20(7):470-5.

38. National Heart L, Blood Institute Acute Respiratory Distress Syndrome Clinical Trials N, Wiedemann HP, Wheeler AP, Bernard GR, Thompson BT, et al. Comparison of two fluidmanagement strategies in acute lung injury. N Engl J Med. 2006;354(24):2564-75. https://doi.org/10.1056/NEJMoa062200.

39. National Heart L, Blood Institute Acute Respiratory Distress Syndrome Clinical Trials N, Rice TW, Wheeler AP, Thompson BT, Steingrub J, et al. Initial trophic vs full enteral feeding in patients with acute lung injury: the EDEN randomized trial. JAMA. 2012;307(8):795-803. https://doi.org/10.1001/jama.2012.137.

40. Needham DM, Dinglas VD, Morris PE, Jackson JC, Hough CL, Mendez-Tellez PA, et al. Physical and cognitive performance of patients with acute lung injury 1 year after initial trophic versus full enteral feeding. EDEN trial follow-up. Am J Respir Crit Care Med. 2013;188(5):567-76. https://doi.org/10.1164/rccm.2013040651OC.

41. Arabi YM, Aldawood AS, Haddad SH, Al-Dorzi HM, Tamim HM, Jones G, et al. Permissive underfeeding or standard enteral feeding in critically ill adults. N Engl J Med. 2015;372(25):2398-408. https://doi.org/10.1056/NEJMoa1502826.

42. Arabi YM, Aldawood AS, Al-Dorzi HM, Tamim HM, Haddad SH, Jones $\mathrm{G}$, et al. Permissive underfeeding or standard enteral feeding in high- and low-nutritional-risk critically ill adults. Post hoc analysis of the PermiT Trial. Am J Respir Crit Care Med. 2017;195(5): 652-62. https://doi.org/10.1164/rccm.201605-1012OC.

43. Compher C, Chittams J, Sammarco T, Nicolo M, Heyland DK. Greater protein and energy intake may be associated with improved mortality in higher risk critically ill patients: a multicenter, multinational observational study. Crit Care Med. 2017;45(2):156-63. https://doi.org/10.1097/CCM.0000000000002083 Study demonstrating the importance of protein in critically ill patients.

44. Gadek JE, DeMichele SJ, Karlstad MD, Pacht ER, Donahoe M, Albertson TE, et al. Effect of enteral feeding with eicosapentaenoic acid, gamma-linolenic acid, and antioxidants in patients with acute respiratory distress syndrome. Enteral Nutrition in ARDS Study Group Crit Care Med. 1999;27(8):1409-20.

45. Rice TW, Wheeler AP, Thompson BT, deBoisblanc BP, Steingrub J, Rock P, et al. Enteral omega-3 fatty acid, gamma-linolenic acid, and antioxidant supplementation in acute lung injury. JAMA. 2011;306(14):1574-81. https://doi.org/10.1001/jama.2011.1435.

46. Singer P, Theilla M, Fisher H, Gibstein L, Grozovski E, Cohen J. Benefit of an enteral diet enriched with eicosapentaenoic acid and gamma-linolenic acid in ventilated patients with acute lung injury. Crit Care Med. 2006;34(4):1033-8. https://doi.org/10.1097/01. CCM.0000206111.23629.0A.

47. Nicolo M, Heyland DK, Chittams J, Sammarco T, Compher C. Clinical outcomes related to protein delivery in a critically ill population: a multicenter, multinational observation study. JPEN J Parenter Enteral Nutr. 2016;40(1):45-51. https://doi.org/10.1177/ 0148607115583675.

48. Reignier J, Boisrame-Helms J, Brisard L, Lascarrou JB, Ait Hssain A, Anguel N, et al. Enteral versus parenteral early nutrition in ventilated adults with shock: a randomised, controlled, multicentre, open-label, parallel-group study (NUTRIREA-2). Lancet. 2018;391(10116):133-43. https://doi.org/10.1016/S01406736(17)32146-3 Study evaluating enteral versus parenteral nutrition in critically ill patients.

Publisher's Note Springer Nature remains neutral with regard to jurisdictional claims in published maps and institutional affiliations. 